\title{
BAYESIAN INFERENCE OF HETEROGENEOUS VISCOPLASTIC MATERIAL PARAMETERS
}

\author{
ElišKa Janouchová*, AnNa KuČEROVÁ \\ Faculty of Civil Engineering, Czech Technical University in Prague, Thákurova 7, Prague, Czech Republic \\ * corresponding author: eliska.janouchova@fsv.cvut.cz
}

\begin{abstract}
Modelling of heterogeneous materials based on randomness of model input parameters involves parameter identification which is focused on solving a stochastic inversion problem. It can be formulated as a search for probabilistic description of model parameters providing the distribution of the model response corresponding to the distribution of the observed data.

In this contribution, a numerical model of kinematic and isotropic hardening for viscoplastic material is calibrated on a basis of experimental data from a cyclic loading test at a high temperature. Five material model parameters are identified in probabilistic setting. The core of the identification method is the Bayesian inference of uncertain statistical moments of a prescribed joint lognormal distribution of the parameters. At first, synthetic experimental data are used to verify the identification procedure, then the real experimental data are processed to calibrate the material model of copper alloy.
\end{abstract}

Keywords: Parameter identification, Bayesian inference, hierarchical modelling, aleatory uncertainty, kinematic and isotropic hardening.

\section{INTRODUCTION}

Reliable predicting behaviour of an investigated system in a computational way requires proper calibration of the corresponding numerical model. This contribution concentrates on modelling heterogeneous materials, where the heterogeneity is captured by randomness of model input parameters. The spatial variability of mechanical properties in heterogeneous materials, e.g. elastic modulus or yield stress, affects behaviour of the investigated structural system under the loading. This can be demonstrated by repeating a laboratory test for an ensemble of specimens made of the same heterogeneous material. Calibration of a heterogeneous material model can be perceived as identification of its parameters' probability distribution when the corresponding model response has the distribution as much as possible similar to the distribution of the observed data. The probabilistic identification of random variables is called a stochastic inversion problem.

Stochastic inversion enables to infer a probability distribution of the unknown model parameters from indirect experimental measurements. In order to make the identification process successful, a proper distinction of occurring uncertainties is required. Essentially, uncertainties can be separated into two basic groups. The first one contains uncertainties connected to a lack of knowledge, it is caused by e.g. measurement errors or a small number of measurements. This uncertainty is called epistemic and it is reducible by any new information. On the other side, there is irreducible stochasticity called aleatory uncertainty or simply variability. It represents an inherent randomness which originates from modelling a data collection e.g. from different locations or times as a random variable [1]. An illustrative examples of uncertainties related to material properties are presented in Figure 1.

Identification procedure of heterogeneous material properties from indirect noisy measurements having a numerical model describing the nonlinear relationship between the unknown inputs and observed outputs is presented in section 2 The principle of the method is a choice of a certain form of probability density function (PDF) of the unknown stochastic model parameters whose statistical moments are considered as uncertain random variables. The probabilistic identification is then concentrated on these so-called hyperparameters of the parameters' distribution which can be inferred in the Bayesian way [2]. This approach has been already applied on a very simple numerical example of a cantilever in the authors' previous work 3 .

This contribution focuses on application of the identification method for a more complicated numerical model leading to the model calibration from real experimental data. In section 3 the material model of kinematic and isotropic hardening for viscoplastic material together with its unknown random input parameters is briefly introduced. Section 4 deals with verification of the proposed identification procedure and following model calibration. The obtained results are then summarized in Section 5 .

\section{Stochastic IDENTIFICATION METHOD}

This section recapitulates the basics of the Bayesian inference and its extension by hierarchical modelling 
Homogeneous material
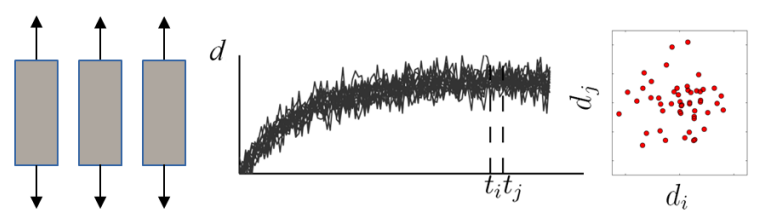

Uncertainty in mean response

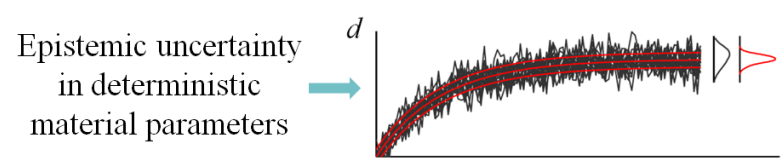

Heterogeneous material

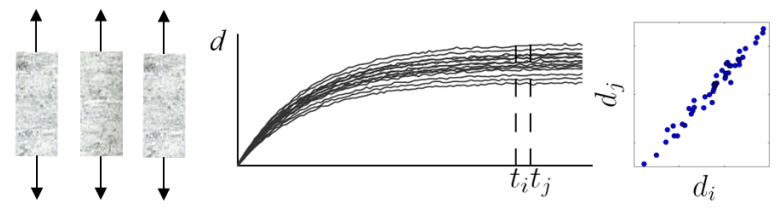

Distribution of response

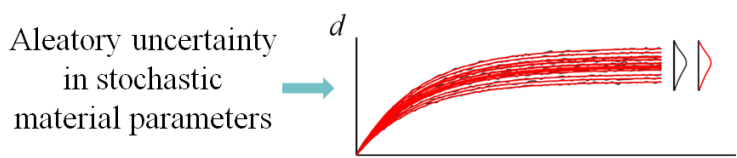

FigURE 1. Uncertainties in properties of a homogeneous and heterogeneous material.

in order to enable an application of this method for identification of aleatory uncertainty presented in the authors' former conference paper [3].

The principal idea of the Bayesian identification is based on a common way of thought when the resulting belief about a random event is given by a combination of all available information [4]. This approach introduces the concept of uncertainty in our subjective knowledge of the identified parameters. The Bayesian inference becomes increasingly popular and more widespread approach to parameter identification providing an elegant solution to this inverse problem by making it well-posed. It allows to estimate values of input parameters together with appropriate uncertainties by combining prior information and experimental measurements. In this case, observations are assumed to be performed for the specific yet unknown values of input parameters and epistemic uncertainty arising from experimental errors and lack of knowledge is reduced with an increasing number of experimental observations.

The Bayesian approach can be extended into multilevel setting which allows to take into account also aleatory uncertainties [5, 6]. While in the classical formulation the model parameters have unknown deterministic values, hierarchical modelling enables to consider the model parameters $\boldsymbol{x}$ as stochastic variables described by some statistical model with uncertain moments. Specifically, the extension is done by introducing new random variables, known as hyperparameters $\boldsymbol{\theta}$ defining the probabilistic specification of the model parameters $\boldsymbol{x}$ [2]. The parameters' prior is then conditional on the hyperparameters $\boldsymbol{\theta}$ with their own prior distribution called hyperprior $p(\boldsymbol{\theta})$, the joint prior distribution of the parameters and hyperparameters has a form

$$
p(\boldsymbol{x}, \boldsymbol{\theta})=p(\boldsymbol{x} \mid \boldsymbol{\theta}) p(\boldsymbol{\theta})
$$

and according to Bayes' rule the corresponding joint posterior distribution up to a normalisation constant is

$$
p(\boldsymbol{x}, \boldsymbol{\theta} \mid \boldsymbol{z}) \propto p(\boldsymbol{z} \mid \boldsymbol{x}, \boldsymbol{\theta}) p(\boldsymbol{x} \mid \boldsymbol{\theta}) p(\boldsymbol{\theta}) .
$$

More specifically, now we have $n$ observations $\boldsymbol{z}_{i}$, each of them is realized for $n$ combinations of input values $\boldsymbol{x}_{i}$ drawn from unknown distribution. Each of these combinations is defined by its prior distribution $f\left(\boldsymbol{x}_{i} \mid \boldsymbol{\theta}\right)$ and according to assumption of observation exchangeability [7] the joint prior distribution is given as

$$
p\left(\boldsymbol{x}_{1}, \ldots, \boldsymbol{x}_{n}, \boldsymbol{\theta}\right)=\left(\prod_{i=1}^{n} f\left(\boldsymbol{x}_{i} \mid \boldsymbol{\theta}\right)\right) p(\boldsymbol{\theta})
$$

and the posterior $p\left(\boldsymbol{x}_{1}, \ldots, \boldsymbol{x}_{n}, \boldsymbol{\theta} \mid \boldsymbol{z}_{1}, \ldots, \boldsymbol{z}_{n}\right)$ as

$$
p(\boldsymbol{x}, \boldsymbol{\theta} \mid \boldsymbol{z}) \propto\left(\prod_{i=1}^{n} f\left(\boldsymbol{z}_{i} \mid \boldsymbol{x}_{i}\right)\right)\left(\prod_{i=1}^{n} f\left(\boldsymbol{x}_{i} \mid \boldsymbol{\theta}\right)\right) p(\boldsymbol{\theta}) .
$$

Our knowledge about the hyperparameters grows by updating based on every new measurement while structure of the model parameters' prior distribution remains unchanged.

\section{NumericAl MODEL}

The identification method is applied to calibration of a numerical model of kinematic and isotropic hardening for copper alloy defined by constitutive equations in Figure 2 .

The available experimental data are depicted in Figure 3 and correspond to 16 repetitions of a straincontrolled cyclic loading test at a high temperature. The material data are represented in relative units: stresses are given as ratios of $r_{r e f}$ and strains as ratios of $\varepsilon_{\text {ref }}$. These reference values are constant throughout this document, so all presented figures are consistent. The experimental errors in each measured point of discretized stress-strain curves are assumed as i.i.d. Gaussian random variables with zero mean values and identic standard deviations. At higher temperatures, the cyclic material behaviour is dominated by the kinematic hardening mechanism and the stress saturation is already reached after a few cycles [8]. The copper alloy does not exhibit a distinct yield limit and its kinematic hardening behaviour is non-linear.

The model parameters to be identified are summarized in Table 1 . Operating with the hardening moduli 


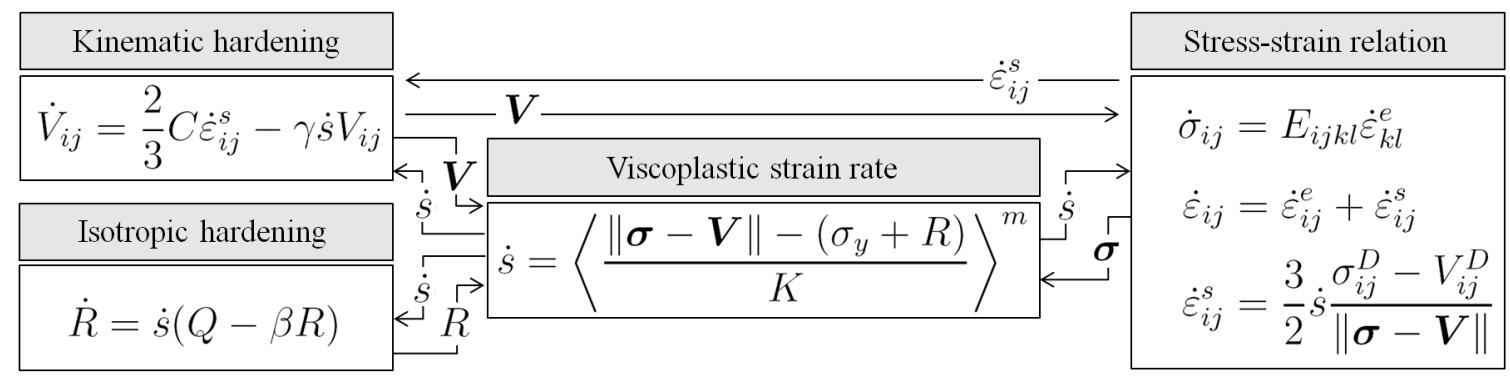

Figure 2. Constitutive equations of the viscoplastic model with non-linear isotropic and kinematic hardening.
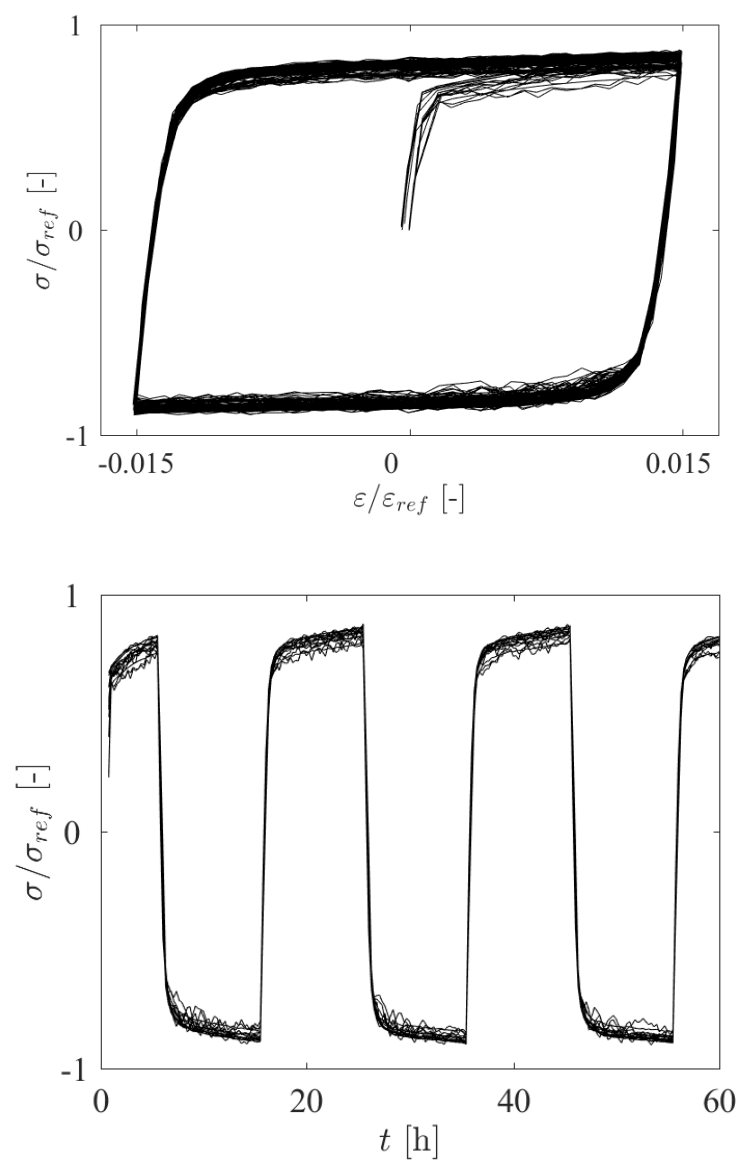

FiguRE 3. Experimental data: stress-strain curves.

$Q$ and $C$, given in equations in Figure 2, is replaced by operating with the saturation values $R_{\infty}=Q / \beta$ and $X_{\infty}=C / \gamma$. The unknown model parameters are assumed as lognormally distributed random variables.

\begin{tabular}{|c|c|c|}
\hline \multicolumn{3}{|c|}{ Parameter Name } \\
\hline & {$[\mathrm{MPa}]$} & Yield stress \\
\hline$V_{\infty}$ & {$[\mathrm{MPa}]$} & Kinematic hardening saturation \\
\hline$\gamma$ & {$[-]$} & Kinematic dynamic recovery constant \\
\hline$R_{\infty}$ & {$[\mathrm{MPa}]$} & Isotropic hardening saturation \\
\hline$\beta$ & {$[-]$} & Isotropic dynamic recovery constant \\
\hline
\end{tabular}

TABLE 1. Model parameters to be identified.
The corresponding mean values and standard deviations are the hyperparameters with uninformative uniform hyperprior.

The information is combined according to Bayes' rule into joint posterior distribution of 90 random variables, specifically, 80 parameters ( 5 model parameters corresponding to each of 16 measurements) and 10 hyperparameters (2 statistical moments of every single parameter's marginal PDF). The Bayesian inference is realized by the Markov chain Monte Carlo (MCMC) method providing posterior samples [9, 10]. The computational effort is reduced by substituting the full numerical model by its polynomial chaos-based approximation, specifically by the Hermite polynomials of standard normal random variables [11, 12. The polynomial coefficients are computed with a help of regression method [13] based on Latin Hypercube sampling 14 from prior parameter distribution.

\section{Model CALibration}

In order to verify the identification method at first, a synthetic experimental data set of 16 model simulations, see Figure 4, is employed. Figure 5 shows histograms corresponding to the simulations' inputs generated from the prescribed lognormal distribution

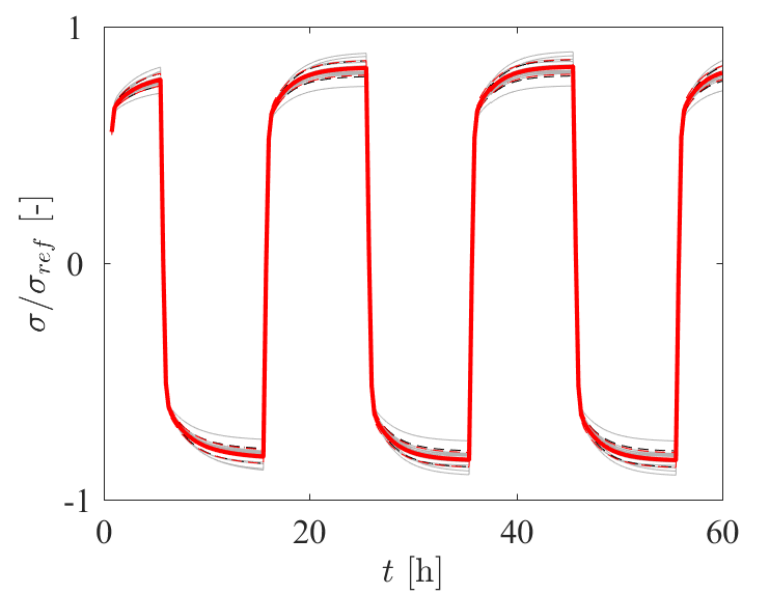

Figure 4. Comparison of synthetic observations (black) with model responses (red) corresponding to the identified parameters' distribution in a form of mean value \pm standard deviation. 

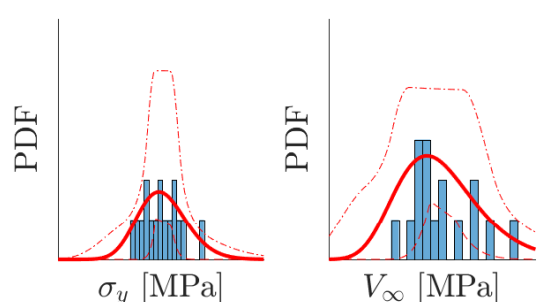

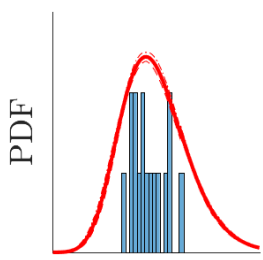

$\gamma[-]$

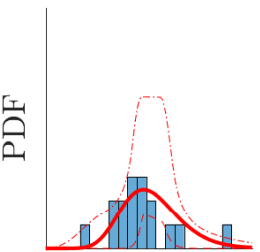

$R_{\infty}[\mathrm{MPa}]$

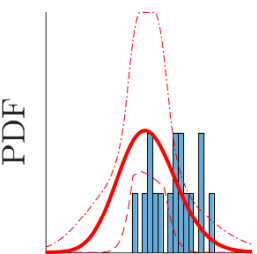

$\beta[-]$

FiguRE 5. Verification: Histograms of synthetic experimental inputs and identified parameters' PDF corresponding to hyperparameters' means accompanied by bounds corresponding to $90 \%$ of hyperparameters' values.
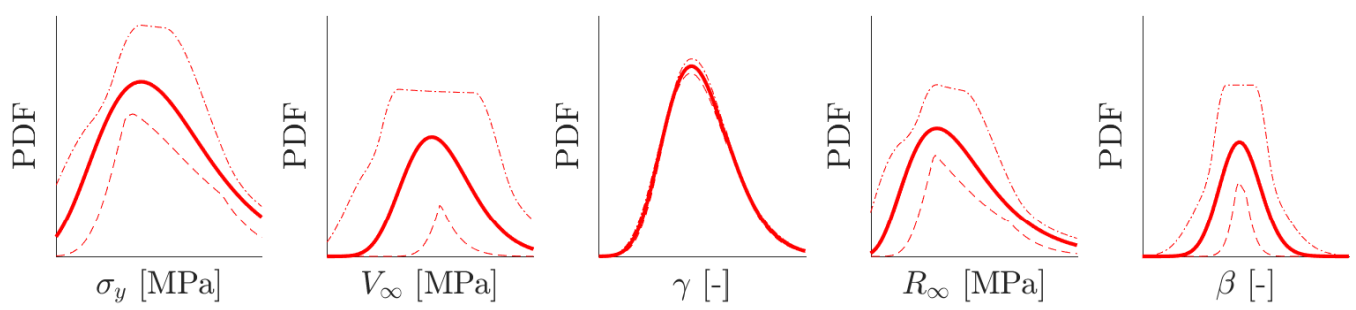

FiguRE 6. Validation: Identified parameters' marginal PDF corresponding to hyperparameters' means accompanied by bounds corresponding to $90 \%$ of hyperparameters' values.

in a comparison with the identified parameter distributions estimated from MCMC posterior samples. The solid lines correspond to parameters' marginal PDFs with identified mean values of hyperparameters representing the aleatory uncertainties in the parameters' values while the dashed lines form an envelope of parameters' PDFs corresponding to $90 \%$ of hyperparameters' values representing the epistemic uncertainties. As expected due to experimental setting, the cyclic material behaviour is dominated by the kinematic hardening mechanism at a high temperature, so while the isotropic hardening parameter $\beta$ is not identified properly, the kinematic hardening parameter $\gamma$ is inferred very precisely and epistemic uncertainties connected to its PDF are reduced significantly.

The identified parameters' PDF corresponding to hyperparameters' mean values is used to quantify aleatory uncertainty in the model response, which is presented in Figure 4. Estimation of the aleatory uncertainty in the model response has a good match with the synthetic experimental data. From this point of view, the results of the proposed identification procedure are satisfactory.

Since the proposed method of model calibration passed the verification process successfully, the model calibration based on the real experimental data follows. The results of model calibration are presented in the same manner as the verification results. The Figure 6 shows the identified most likely marginal parameters' PDFs with depicted epistemic uncertainties. Now, it is not possible to compare the results on a level of model parameters' values, but the epistemic uncertainties are reduced the most in the case of the parameter $\gamma$ again due to the experimental setup. The aleatory uncertainty caused by heterogeneous character of the modelled material is sufficiently captured, see Figure 7 presenting comparison of experimental data and model responses corresponding to the identified parameters' PDF.

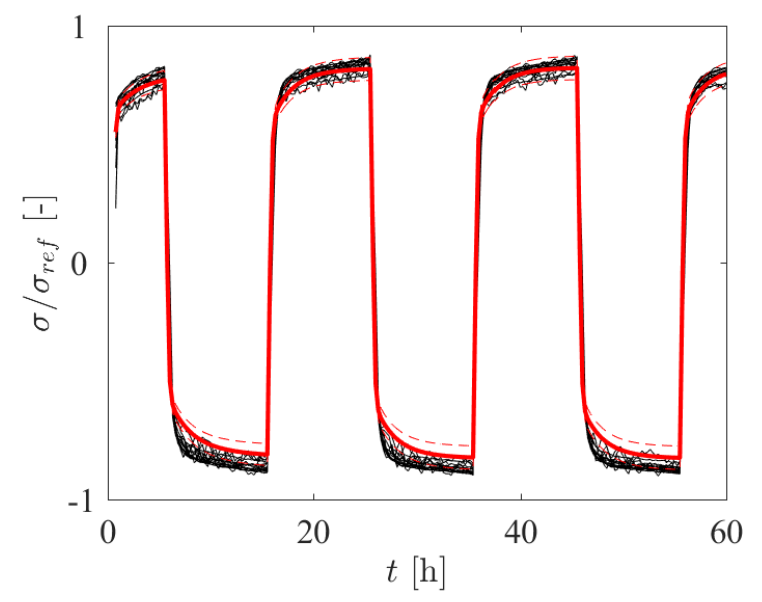

FiguRE 7. Comparison of experimental observations (black) with model responses (red) corresponding to the identified parameters' distribution in a form of mean value \pm standard deviation.

\section{Conclusions}

The multilevel setting of the Bayesian inference provides an elegant solution for stochastic inversion problems. This efficient tool enables to separate epistemic uncertainties and inherent variability, which is fundamental for successful parameter identification of heterogeneous material model from indirect noisy measurements. The major disadvantage of this method is the requirement of assuming some specific type of parameters' PDF which affects the result essentially and 
it can be problematic for example in the case of phenomenological parameters. The formulated posterior probability distribution can be gained in a form of independent samples by the Markov chain Monte Carlo method, however the computational demands increase significantly with a number of measurements, so this approach is suitable especially for small experimental data sets.

In this contribution, the material model of kinematic and isotropic hardening for copper alloy is successfully calibrated on a basis of the experimental data set of 16 repetitions of a strain-controlled cyclic loading test at a high temperature. At first, identification of aleatory as well as epistemic uncertainties with a help of the Bayesian inference is presented for the case of synthetically generated experimental data. After the successful verification of the identification procedure, the real experimental data are processed in the same way to calibrate the numerical model. Due to the experimental setup, when kinematic hardening of the material plays the main role, the parameters of kinematic hardening are identified more precisely than the parameters of isotropic hardening. In order to identify all the parameters more accurately, some more data from another type of experiment have to be available to obtain the necessary additional information.

\section{ACKNOWLEDGEMENTS}

This outcome was financially supported by the Czech Science Foundation, project No. 16-11473Y, and the Grant Agency of the Czech Technical University in Prague, grant No. SGS18/036/OHK1/1T/11.

\section{REFERENCES}

[1] W. L. Oberkampf, S. M. DeLand, B. M. Rutherford, et al. Error and uncertainty in modeling and simulation. Reliability Engineering 85 System Safety 75(3):333-357, 2002.

[2] A. Gelman, J. B. Carlin, H. S. Stern, D. B. Rubin. Bayesian data analysis. Chapman \& Hall/CRC, druhé edn., c2004.

[3] E. Janouchová, A. Kučerová, J. Sýkora. Bayesian updating of aleatory uncertainties in heterogeneous materials. In Modern Methods of Experimental and Computational Investigations in Area of Construction II, vol. 1144 of Advanced Materials Research, pp. 136-141. Trans Tech Publications, 2017.
[4] T. Bayes, R. Price. An essay towards solving a problem in the doctrine of chances. by the late rev. $\mathrm{mr}$. bayes, f. r. s. communicated by mr. price, in a letter to john canton, a. m. f. r. s. Philosophical Transactions (1683-1775) 53:370-418, 1763.

[5] J. B. Nagel, B. Sudret. A unified framework for multilevel uncertainty quantification in bayesian inverse problems. Probabilistic Engineering Mechanics 43:68-84, 2016.

[6] I. Behmanesh, B. Moaveni, G. Lombaert, C. Papadimitriou. Hierarchical bayesian model updating for structural identification. Mechanical Systems and Signal Processing 64:360-376, 2015.

[7] D. Draper, J. S. Hodges, C. L. Mallows, D. Pregibon. Exchangeability and data analysis. Journal of the Royal Statistical Society Series A (Statistics in Society) pp. 9-37, 1993.

[8] W. Schwarz, S. Schwub, K. Quering, et al. Life prediction of thermally highly loaded components: modelling the damage process of a rocket combustion chamber hot wall. CEAS Space Journal 1(1-4):83, 2011.

[9] J. C. Spall. Estimation via markov chain monte carlo. IEEE Control Systems Magazine 23(2):34-45, 2003.

[10] Y. M. Marzouk, H. N. Najm, L. A. Rahn. Stochastic spectral methods for efficient bayesian solution of inverse problems. Journal of Computational Physics 224(2):560-586, 2007.

[11] N. Wiener. The homogeneous chaos. American Journal of Mathematics 60(4):897-936, 1938.

[12] D. Xiu, G. E. Karniadakis. The wiener-askey polynomial chaos for stochastic differential equations. SIAM Journal on Scientific Computing 24(2):619-644, 2002.

[13] G. Blatman, B. Sudret. An adaptive algorithm to build up sparse polynomial chaos expansions for stochastic finite element analysis. Probabilistic Engineering Mechanics 25(2):183-197, 2010.

[14] R. L. Iman, W. Conover. Small sample sensitivity analysis techniques for computer models. with an application to risk assessment. Communications in statistics-theory and methods 9(17):1749-1842, 1980. 